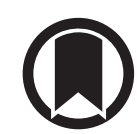

CrossMark

\title{
Assessing self-medication for obstructive airway disease during COVID-19 using Google Trends
}

\author{
To the Editor:
}

COPD is a leading cause of morbidity and mortality worldwide, affecting approximately 294 million people worldwide, and accounting for over 3 million deaths annually. Similarly, asthma affects approximately 268 million people worldwide, leading to an enormous health burden [1]. With the outbreak of the coronavirus disease 2019 (COVID-19) pandemic, the Global Initiative for Chronic Obstructive Lung Disease (GOLD) set a main emphasis on recommendations focused on the regular use of bronchodilator maintenance therapy, and the Global Initiative for Asthma (GINA) guidelines 2020 explicitly state that inhaler therapy, especially the maintenance therapy with inhaled corticosteroids (ICS), should not be interrupted during the pandemic $[2,3]$.

Coinciding with the outbreak of COVID-19 in Austria with its first major hot-spot in the Alpine state of Tyrol (inhabitants: 757852; capital: Innsbruck), and during its associated lockdown (18 March to 7 April 2020), we experienced a dramatic decrease in COPD-related hospitalisations at the Department of Internal Medicine, Innsbruck Medical University. Therefore, using Google's search engine data analysis tool Google Trends (https://trends.google.com/trends/) [4], we aimed to investigate whether patients suffering from COPD and/or asthma may have consulted the internet for behavioural and self-medication advice.

As shown in figure 1a, we observed a marked reduction in daily hospitalisations for COPD and asthma during the COVID-19 outbreak between February and April 2020 at our Department of Internal Medicine, when compared to the years 2017-2019. In line, we observed a drop in hospitalisations for non-COVID-19 pneumonia, whereas COVID-19 associated hospitalisation rates dramatically increased, peaking on March 27, 2020. The yearly seasonal peak in influenza-associated admissions between November and the end of March was significantly shortened this year $(-19 \pm 8$ days compared to the mean duration in 2015-2019), which is consistent with the observed shortening of the influenza pandemic in the Northern hemisphere due to the lockdown and social-distancing measures reported by FluNet [5]. The World Health Organization-based FluNet also states that the ongoing COVID-19 pandemic may have influenced health-seeking behaviour. Pre-existing conditions constituting a major risk for COVID-19 include asthma, COPD, hypertension, diabetes and cancer [6]. Importantly, with the outbreak of the COVID-19 pandemic, much has been discussed about ACE inhibitors, hypertension and associated risks for experiencing a severe course of COVID-19, and apparently less about other risk factors, including respiratory diseases [7].

On 25 August 2020, we queried Google Trends and downloaded the data. Among the synonymous search terms for "COVID-19" suggested by Google Trends worldwide, the search term with the highest relative search volume (RSV) was by far "coronavirus" and thus used in the current analysis ("coronavirus"> "COVID-19"> "SARS"). When querying for the topics "chronic obstructive pulmonary disease" ("COPD") and "asthma" we observed a significant rise in RSV from the end of February to the beginning of April 2020 when compared to the past 3 years (asthma RSV $69 \pm 19$ versus $36 \pm 1 \mathrm{p}<0.01$; COPD RSV $28 \pm 6$ versus $19 \pm 1 \mathrm{p}<0.01$ ). Like SHARMa and SHARMa [8], we conducted a subanalysis of individual countries, restricting the analysis to countries with sufficient internet traffic, well-documented COVID-19 burden, and comparable lockdown measures (USA, UK, Germany, France, Italy, and Spain).

@ERSpublications

Google Trends reveals public information-seeking for self-medication in patients with obstructive respiratory disease during the COVID-19 outbreak, illustrating the urgent need to implement digital health support in patients with respiratory disease https://bit.ly/2GRccSU

Cite this article as: Sahanic S, Boehm A, Pizzini A, et al. Assessing self-medication for obstructive airway disease during COVID-19 using Google Trends. Eur Respir J 2020; 56: 2002851 [https://doi.org/10.1183/ 13993003.02851-2020]. 

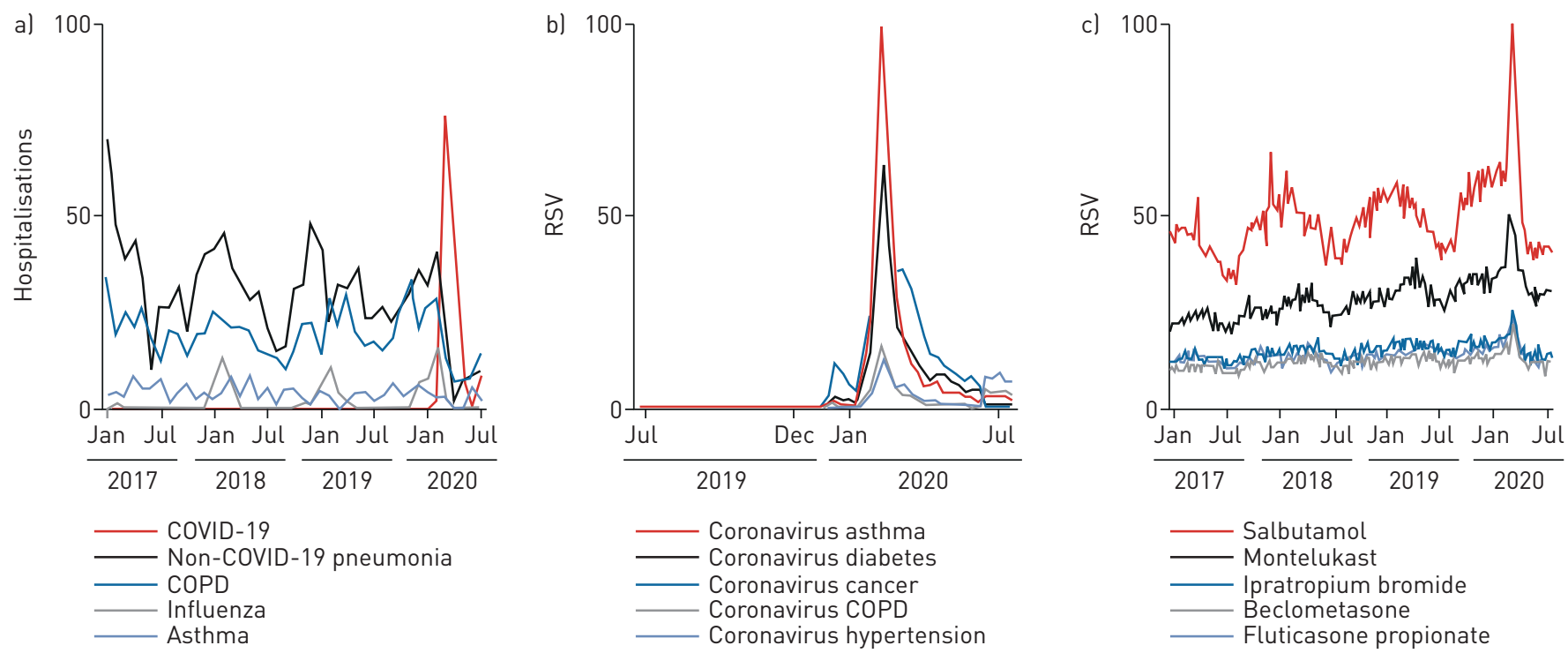

FIGURE 1 a) Daily hospitalisation rates for coronavirus disease 2019 (COVID-19) and main respiratory conditions leading to hospital admission at the Department of Internal Medicine, Medical University of Innsbruck, Austria between 2017 and 2020. b) Worldwide relative search volume (RSV) of pre-existing conditions with a high risk for a severe course of COVID-19 associating with the term "coronavirus". c) Worldwide RSV of medications used by asthma and COPD patients between 2017 and 2020. RSV ranges from 1 to 100, representing search interest relative to the peak popularity for the used search term. An RSV value of 100 indicates peak popularity and a score of 0 indicates that the term is below 1 percent of its peak popularity.

Consistent results were observed when analysing "asthma" and "COPD", with the exception of Italy showing no relevant elevation in "COPD” RSV during March 2020 (data not shown).

Comparing the search terms "coronavirus asthma" and "coronavirus COPD" with "coronavirus hypertension", "coronavirus diabetes" and "coronavirus cancer", we observed worldwide highest RSV for "coronavirus asthma" followed by "coronavirus diabetes" and "coronavirus cancer" (figure 1b). Surprisingly, "coronavirus hypertension" ranked fourth together with "coronavirus COPD". When exploring the health-seeking behaviour of patients affected by asthma and/or COPD during the COVID-19 outbreak, we focused on those therapeutic approaches with the highest RSV worldwide and that were thus comparable. As shown in figure 1c, Google Trends analysis revealed highest RSV for topics "salbutamol", "montelukast", "ipratropium bromide", "beclometasone", and "flucticasone propionate", paralleling the worldwide COVID-19 outbreak. No consistent results could be observed when analysing the RSVs of these medications in individual countries, probably reflecting the vast number of available products and country-dependent preferences (data not shown).

To summarise, our analysis revealed highest search volumes for asthma and asthma-associated medications, despite other risk factors like hypertension having been largely debated in the media [9]. In this context, asthma patients may represent a clearly younger collective than patients suffering from hypertension, thus potentially having easier access to the internet. Age, together with a more intuitive access to web-based search and advice, may at least in part explain why the RSV for "asthma" and "coronavirus asthma" were found to be markedly higher than the RSV for "COPD" and "coronavirus COPD". On the other hand, it may also simply reflect the poor global awareness for COPD, as shown in our previous report, where COPD was only ranked eighth in RSV among the top 10 causes of death in high-income countries [4].

Interestingly, when focusing on inhaler therapy, search terms with highest RSV and thus suitable for our Google Trends analysis encompassed mainly relievers, i.e. short-acting $\beta$-agonists, and short-acting muscarinic antagonists, followed by ICS, indicating patients were mainly seeking for advice in case of an exacerbation and potentially SARS-CoV-2 infection. Considering the GINA guidelines, in which the authors explicitly state that asthma treatment should no longer be based solely on short-acting bronchodilators, our data clearly indicates a failure in reaching asthma patients with respective fundamental changes in therapy [10]. Interestingly, no usable data associated with the COVID-19 pandemic could be retrieved from Google Trends regarding maintenance therapy with long-acting $\beta$-agonists and long-acting antimuscarinics, or a combination of both, the latter representing a cornerstone in the prevention of COPD exacerbations, and therefore reflecting scarce knowledge about preventive therapeutic approaches within the population affected by COPD. 
Although the data presented herein will help to understand trending needs of patients with obstructive respiratory disease, we have to acknowledge some limitations. First, Google Trends does not provide insights into the characteristics of individuals contributing to search volumes. Second, search volumes may be increased during events or conditions with large media coverage. Finally, in a population with poor knowledge about a disease, Google Trends might underestimate the real-world burden of the investigated conditions per se, and therefore our findings will require validation through epidemiological studies.

In summary, both social distancing and lockdown measures, together with increased self-medication and digital care-seeking, may have reduced hospital admission rates in patients affected by obstructive respiratory disease during the COVID-19 outbreak in early 2020. However, our data urge further improvement in implementing digital health monitoring and advice in patients affected by asthma and COPD.

Sabina Sahanic ${ }^{1,2}$, Anna Boehm $\oplus^{1,2}$, Alex Pizzini $\oplus^{1}$, Thomas Sonnweber $\odot^{1}$, Magdalena Aichner ${ }^{1}$, Guenter Weiss ${ }^{1}$, Judith Loeffler-Ragg ${ }^{1}$ and Ivan Tancevski $\circledast^{1}$

${ }^{1}$ Dept of Internal Medicine II, Infectious Diseases, Pneumology, Rheumatology, Medical University of Innsbruck, Innsbruck, Austria. ${ }^{2}$ S. Sahanic and A. Boehm contributed equally to this manuscript.

Correspondence: Alex Pizzini, Dept of Internal Medicine II, Infectious Diseases, Pneumology, Rheumatology, Medical University of Innsbruck, Anichstrasse 35, 6020 Innsbruck, Austria. E-mail: alex.pizzini@i-med.ac.at

Received: 21 July 2020 | Accepted after revision: 7 Sept 2020

Author contributions: S. Sahanic, A. Boehm, A. Pizzini, T. Sonnweber, M. Aichner, G. Weiss, J. Loeffler-Ragg and I. Tancevski conceived and designed the study. S. Sahanic and A. Boehm drafted the manuscript and collected the data. S. Sahanic, A. Boehm, A. Pizzini, J. Loeffler-Ragg and I. Tancevski analysed and interpreted the data. S. Sahanic, A. Boehm, A. Pizzini, T. Sonnweber, M. Aichner, G. Weiss, J. Loeffler-Ragg and I. Tancevski revised and approved the manuscript.

Conflict of interest: None declared.

\section{References}

$1 \quad$ GBD Chronic Repiratory Disease Collaborators. Prevalence and attributable health burden of chronic respiratory diseases, 1990-2017: a systematic analysis for the Global Burden of Disease Study 2017. Lancet Repir Med 2020; 8: 585-596.

2 Global Initiative for Chronic Obstructive Lung Disease (GOLD). GOLD Covid-19 Guidance. 2020. https:// goldcopd.org/gold-covid-19-guidance/ Date last accessed: 20 July 2020.

3 Global Initiative for Asthma. Global Strategy for Asthma Management and Prevention. GINA, 2020. Available from www.ginasthma.org.

4 Boehm A, Pizzini A, Sonnweber T, et al. Assessing global COPD awareness with Google Trends. Eur Respir J 2019; 53: 1900351.

5 World Health Organization (WHO). FluNet. www.who.int/influenza/gisrs_laboratory/flunet/en/ Date last updated: 17 August 2020. Date last accessed: 23 August 2020.

6 Williamson EJ, Walker AJ, Bhaskaran K, et al. OpenSAFELY: factors associated with COVID-19 death in 17 million patients. Nature 2020; 584: 430-436.

7 Fosbøl EL, Butt JH, Østergaard L, et al. Association of angiotensin-converting enzyme inhibitor or angiotensin receptor blocker use with COVID-19 diagnosis and mortality. JAMA 2020; 324: 168-177.

8 Sharma M, Sharma S. The rising number of COVID-19 cases reflecting growing search trend and concern of people: a Google Trend analysis of eight major countries. J Med Syst 2020; 44: 117.

9 Kanwal A, Agarwala A, Warsinger Martin L, et al. COVID-19 and Hypertension: What We Know and Don't Know. www.acc.org/latest-in-cardiology/articles/2020/07/06/08/15/covid-19-and-hypertension Date last updated: 6 June 2020.

10 Reddel HK, FitzGerald JM, Bateman ED, et al. GINA 2019: a fundamental change in asthma management. Eur Respir J 2019; 53: 1901046. 